


\title{
Apalancamiento financiero. Efectos sobre el rendimiento económico y financiero de los proyectos de inversión 1
}

Financial leverage. Effects on the economic and financial performance of investment projects

\author{
Juan Gallardo Cervantes \\ Facultad de Economía de la UnAM \\ Correo electrónico: gallardocervantesjuan@yahoo.co.uk
}

(Recibido: 24/04/2018. Aceptado para publicación: 30/08/2018)

DOI: 10.22201/fe.24484962e.2018.v7n12.a4

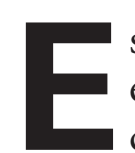
ste trabajo tiene como objetivo principal señalar de manera simple y profusa los efectos que tiene el apalancamiento financiero sobre el rendimiento del capital social invertido en un proyecto de inversión o en una organización, así como sobre los diversos indicadores y estados financieros empleados para determinar la evaluación económica y financiera de dicho proyecto de inversión u organización.

Partamos de la siguiente situación. Hace justamente un año, Gianluca Riccolino, un italiano avecindado en Chipilo, Puebla, comenzó un negocio de productos lácteos el cual ha prosperado bastante bien o al menos mejor de lo que Gianluca esperaba. El producto fabricado por Gianluca es un queso del tipo provolone y en su elaboración se requieren diez litros de leche para fabricar 1.5 kilos de queso, además de otros ingredientes "secretos" que sólo Gianluca conoce. Para iniciar este negocio, el propietario tuvo que hacer un desembolso en efectivo de \$1 844980.00 mediante el cual adquirió los activos fijos, diferidos y el capital de trabajo requerido para poner en marcha el negocio.

Hace un mes, Gianluca recibió noticias procedentes de Italia en el sentido de que su presencia urge para resolver un problema de una herencia. Por tal motivo, Gianluca hizo planes para regresar a Italia, sin la intención de volver a México, por lo cual elaboró un pequeño análisis financiero sobre su incipiente negocio a fin de buscar un socio o un comprador que continúe al frente del mismo.

\footnotetext{
1 Este trabajo fue financiado con recursos del Programa de Apoyo a Proyectos para la Innovación y Mejoramiento de la Enseñanza (PAPIME) que coordina la Dirección General de Asuntos del Personal Académico (DGAPA) de la UNAM, mediante el proyecto número PE313418.
} 
Para el efecto anterior, Gianluca, quien es tu amigo y sabe que estudias economía, te contrata para que le ayudes a recopilar la siguiente información financiera sobre el negocio y le ayudes a tomar una decisión sobre el traspaso o venta del mismo, determinando primeramente el rendimiento que Gianluca obtuvo durante ese año:

1. La inversión original requerida para iniciar el procesamiento y comercialización del queso provolone fue realizada hace un año por una suma total de \$1 844 980.00; de los cuales $\$ 875000.00$ fueron para el equipo de proceso; \$289 000.00 para el equipo de transporte; $\$ 12000.00$ más se usaron para el pago de diversos permisos; otros \$25 500.00 para pagar los honorarios del notario que hizo el protocolo de la constitución jurídica; \$389000.00 fueron para capital de trabajo y el resto, $\$ 254480.00$, correspondieron al Impuesto al Valor Agregado (IVA) pagado por los activos adquiridos. El siguiente cuadro muestra el desglose de la inversión requerida mostrando tres columnas: la primera se refiere al importe pagado por Gianluca al momento de la adquisición, la segunda se refiere al IVA pagado, mismo que podrá recuperar (acreditar) cuando haga la declaración de impuestos, y la tercera columna se refiere a los importes que fiscal y financieramente se deben considerar cuando los activos se deprecien o cuando se requiera de algún tipo de crédito.

2. Las ventas anuales de queso provolone fueron por 147300 kilos, parte de las cuales se hicieron al mayoreo y otras al menudeo a precios diferenciales en los siguientes términos: 12540 kilos se vendieron a $\$ 100.00$ por kilo; 29356 kilos más fueron colocados a $\$ 90.00$ por kilo y el resto, 105404 kilos, se vendieron a $\$ 80.00$ por kilo. De esta manera, el precio promedio al que se vendió el queso fue de $\$ 83.46$ por kilo.

3. El costo directo de fabricar y comercializar el queso es el más importante dentro de la estructura de costos y representa aproximadamente el $67 \%$ del precio de venta, es decir, asciende a $\$ 56.08$ por kilo.

4. Los gastos de operación anuales fueron por \$2 569500.00 , mismos que se aglutinaron en dos conceptos: los gastos erogados en efectivo y los gastos de tipo virtual. Los primeros suman $\$ 2406000.00$, incluido un sueldo que el economista asignó a Gianluca como costo de oportunidad por la administración del negocio. Mientras que los gastos virtuales por conceptos de depreciación de los activos fijos (equipos de proceso y transporte) y la amortiza-

ACTIVOS REQUERIDOS POR LA PLANTA DE QUESO PROVOLONE (\$)

\begin{tabular}{|c|c|c|c|}
\hline Conceptos de activo & Importe total & IVA pagado & Importe fiscal \\
\hline Equipo de proceso & 1015000.00 & 140000.00 & 875000.00 \\
\hline Equipo de transporte & 335240.00 & 46240.00 & 289000.00 \\
\hline Permisos & 13920.00 & 1920.00 & 12000.00 \\
\hline Constitución jurídica & 29580.00 & 4080.00 & 25500.00 \\
\hline Capital neto de trabajo & 451240.00 & 62240.00 & 389000.00 \\
\hline Importe total & 1844980.00 & 254480.00 & 1590500.00 \\
\hline
\end{tabular}


CARGO ANUAL POR DEPRECIACIÓNY AMORTIZACIÓN DE LA PLANTA DE QUESO PROVOLONE (\$)

\begin{tabular}{|l|c|c|c|}
\hline Conceptos de activo & Importe fiscal & $\begin{array}{c}\text { Tasas de deprecia- } \\
\text { ción o amortización }\end{array}$ & $\begin{array}{c}\text { Cargo por deprecia- } \\
\text { ción o amortización }\end{array}$ \\
\hline Equipo de proceso & 875000.00 & $10 \%$ & 87500.00 \\
Equipo de transporte & 289000.00 & $25 \%$ & 72250.00 \\
Permisos & 12000.00 & $10 \%$ & 1200.00 \\
Constitución jurídica & 25500.00 & $10 \%$ & 2550.00 \\
Capital neto de trabajo & 389000.00 & $0 \%$ & 0.00 \\
Gastos virtuales anuales & & & 163500.00 \\
\hline
\end{tabular}

ción de los activos intangibles (permisos y constitución jurídica), de acuerdo con las tasas provistas por la Ley del Impuesto Sobre la Renta vigente, fueron por $\$ 163$ 500.00, tal como se puede observar en el cuadro de arriba.

Considerando la depreciación y amortización que sufrieron los activos al final de este primer año de actividades, el valor en libros de estos activos, al final del año de operación de la fábrica, fue de \$1 004250.00 y será considerado como el valor de rescate, asumiendo que se liquidaran todos los activos al final del primer año de operaciones. Cabe notar que el gasto de operación es más o menos fijo y que dicho gasto representa el riesgo de operación de la fábrica de queso.

Con esta información se procedió a hacer los estados e indicadores financieros, que se presentan más abajo, de los cuales proceden las siguientes conclusiones, las cuales son parte de los criterios que Gianluca debe conocer al momento de vender o traspasar su negocio:

1. En primer lugar, se advierte que este negocio genera un margen de 33 centavos por cada peso que se obtiene de ingreso por ventas y que el negocio alcanza el punto de equilibrio cuando sus ingresos por venta son de $\$ 7786646.00$, esto es, con el $63.2 \%$ de las ventas realizadas. Obviamente, al lograr el punto de equilibrio con ese porcentaje, queda claro que las utilidades netas del negocio las genera el $36.8 \%$ de las ventas excedentes al punto de equilibrio, las cuales, como puede verse en el siguiente Estado de Resultados, alcanzan poco más de 1.2 millones de pesos al año.

ESTADO DE RESULTADOS PROFORMA CUANDO NO HAY CRÉDITO (\$)

\begin{tabular}{|lc|}
\hline Ingresos por ventas & 12329010.00 \\
Costo de lo vendido & 8260584.00 \\
Utilidad bruta & 4068426.00 \\
Gastos de operación & 2569500.00 \\
$\quad$ Gastos erogados en efectivo & 2406000.00 \\
$\quad$ Gastos virtuales & 163500.00 \\
Utilidad de operación & 1498926.00 \\
Impuesto sobre la renta (30\%) & 449677.80 \\
Participacion de los trabajadores en las utilidades (10\%) & 149892.60 \\
Utilidad neta & 899355.60 \\
\hline
\end{tabular}


2. Teniendo en cuenta que este estado financiero tiene como propósito principal el cálculo de los compromisos fiscales y laborales - Impuesto Sobre la Renta (ISR) y Participación de los Trabajadores en las Utilidades (PTU) - fue necesario hacer otro estado financiero mediante el cual se determinará el flujo neto de efectivo para hacer la evaluación del proyecto, dicho estado financiero se llama "Estado de Fuentes y Usos del Efectivo", el cual incluye partidas que se omiten en el Estado de Resultados, tales como el IVA acreditable, los gastos virtuales (que por no representar erogaciones de efectivo su importe se encuentra en caja y bancos) y el valor de rescate de los activos al final de ese primer año de operaciones.

El Estado de Fuentes y Usos del Efectivo proporciona el flujo neto de efectivo que se requiere para determinar la rentabilidad del proyecto. En este caso, dado que el financiamiento de los activos se hizo exclusivamente con capital social, el rendimiento se denominará "económico" para diferenciarlo de aquel que si incluye apalancamiento financiero dentro de la estructura financiera empleada en el financiamiento de los activos, al que llamaremos "financiero".

El flujo neto de efectivo anual está constituido por las salidas de efectivo más las entradas de efectivo durante el horizonte de planeación; así, para el caso de esta empresa, el flujo neto de efectivo sería de -\$1 844 980.00 para el año 0 y de \$2 321585.00 para el año 1. El signo solamente se emplea para identificar si se trata de una salida (negativo) o de una entrada (positivo), como puede observarse en el cuadro de abajo.

El cálculo de la rentabilidad se hizo mediante la tasa interna de rendimiento (TIR), que en este caso denominaremos TIRE (económica) para indicar que la estructura financiera empleada en la financiación de los activos no incluye créditos de ningún tipo. El cálculo de este indicador no requiere de una tasa de descuento preestablecida, sino que el indicador representa cuál es el rendimiento anual promedio a interés compuesto, y dicho rendimiento será considerado como el costo de oportunidad que tienen los recursos

ESTADO DE FUENTESY USOS DEL EFECTIVO PROFORMA CUANDO NO HAY CRÉDITO (\$)
Fuentes de efectivo
Capital social
1844980.00
Utilidades netas
899355.60
Gastos virtuales
163500.00
IVA acreditable
254480.00
Valor de rescate
1004250.00
Total de fuentes de efectivo
1844980.00
2321585.60
Usos del efectivo
Activos fijos
1164000.00
Activos diferidos
37500.00
Capital neto de trabajo
389000.00
IVA pagado
254480.00
Total de usos de efectivo
1844980.00
0.00
Flujo neto de efectivo
0.00
2321585.60 
CÁLCULO DE LA TASA INTERNA DE RENDIMIENTO ECONÓMICA

\begin{tabular}{|l|c|r|r|r|r|}
\hline Años & Fne & FA al 25\% & Fne actualizado & FA al 26\% & \multicolumn{1}{c|}{ Fne } \\
\hline 0 & -1844980.00 & 1.0000 & -1844980.00 & 1.0000 & -1844980.00 \\
1 & 2321585.60 & 0.8000 & 1857268.50 & 0.7937 & 1842528.30 \\
Valor actual neto & & & 12288.50 & & -2451.70 \\
\hline
\end{tabular}

de Gianluca cuando éste trate de hacer nuevas inversiones o cuando decida vender, traspasar o arrendar el negocio.

Para su cálculo se requiere de una tabla como la que se muestra arriba, cuyas columnas se explicarán a continuación.

Los factores de actualización (FA) se calculan mediante la siguiente fórmula:

$$
F A=\frac{1}{(1+i)^{n}}
$$

donde la $i$ representa la tasa de descuento o actualización y la $n$ el periodo de tiempo (en este caso, el año para el cual se desea calcular el factor).

Los factores de actualización representan el valor actual de cada peso o unidad monetaria recibida en el futuro. Así, 0.8000 significa que un peso que se recibe al final del primer año es equivalente a 80 centavos de hoy, cuando la tasa de descuento es del $25 \%$ anual. Si la tasa aumenta, el factor disminuye; si la tasa disminuye, el factor aumenta.

El flujo actualizado de cada año es simplemente el producto del flujo neto de efectivo (Fne) por el factor de actualización. El valor actual neto es la suma vertical, y como puede verse dicho valor es positivo cuando se usa una tasa del 25\%, mientras que se hace negativo cuando se usa una tasa del $26 \%$. Dado que la Tire es la tasa que hace que el valor actual neto sea cero, entonces podemos deducir que dicha TIRE es mayor a $25 \%$, pero menor que $26 \%$.
En este caso, dicha TIRE resultó ser de $25.8337 \%$, esto es, que el negocio de queso provolone generó un rendimiento anual cercano al 26\%. Este indicador permitirá a Gianluca tener una idea más apropiada acerca de lo que deberá pedir por su negocio en caso de venderlo o traspasarlo. O en otras palabras, la tasa interna de rendimiento servirá para que Gianluca valore su negocio en términos de un número de veces dicha TIRE.

La TIRE se calculó mediante la fórmula:

$$
\begin{gathered}
\text { TIRE }=25 \%+\frac{(26-25)(12288.50)}{(12288.50+2451.70)} \\
\text { TIRE }=25 \%+0.8337 \%
\end{gathered}
$$

Hasta aquí toda va muy bien para Gianluca, pero surge la siguiente pregunta: ¿cuál hubiera sido la tasa de rendimiento anual si el financiamiento de los activos incluyera pasivos financieros? Gianluca no es afecto a pedir préstamos, pero usted lo anima a que vea qué sucede con la rentabilidad del capital social en caso de que él hubiese tomado un crédito, el cual represente alguna proporción del importe total de los activos, digamos ¿cuál sería el rendimiento del negocio de queso provolone si el $20 \%$ del valor de los activos se hubiese financiado mediante un crédito y el resto mediante capital social?

Para comenzar, es necesario saber o conocer las reglas de operación del intermediario financiero que otorgaría el crédito. Asumamos que dichas reglas están definidas en los siguientes términos: 
- El intermediario financiero maneja dos líneas de crédito: una de créditos refaccionarios y otra de créditos de avío. La primera está destinada al financiamiento de activos fijos y diferidos, mientras que la segunda únicamente para capital neto de trabajo.

- Las líneas de crédito no aplican para financiar ningún tipo de terreno ni para el pago de impuestos, éstos deben ser financiados con recursos propios. Asimismo, el importe máximo de cualquier tipo de crédito no debe exceder del $80 \%$ del importe financiable, es decir, sin incluir terrenos ni impuestos.

- Las tasas de interés anuales son tasas anuales equivalentes (TAE), ya que tanto intereses como principal se pagan mensualmente sobre saldos insolutos (deudores), y son las mismas tanto para los créditos refaccionarios como para los de avío, siendo dicha TAE del $25 \%$.

- Los plazos máximos para los distintos tipos de créditos son de cinco años para los refaccionarios y de un año para los de avío.

Usted considera que dado que el negocio sólo duró un año, y siendo que las tasas son las mismas para cualquier línea de crédito, entonces la estructura financiera para el fi- nanciamiento de los activos que son objeto de crédito sería de la siguiente manera:

- Un crédito por el $20 \%$ del importe fiscal del siguiente cuadro (que no incluye IVA ni terrenos), el cual suma \$318 100.00, esto es, \$1 $590500.00 \times 0.2$.

- Un capital social para cubrir el resto de la adquisición de activos por \$1 526880.00 .

- La estructura financiera suma el mismo importe de \$ 1844980.00 que se requiere para que la fábrica inicie operaciones.

Con esta información, la empresa obtendría un crédito por $\$ 318100.00$ y pagaría anualmente al intermediario financiero una suma de $\$ 79525.00$ por concepto de gastos financieros, es decir, el 25\% de \$318 100.00. Estos gastos serán adicionales al gasto de operación que ya tiene la organización, y es necesario decir que así como existe un riesgo de operación del negocio, el cual deriva del gasto de operación, de igual manera el gasto financiero procedente del crédito representa un riesgo adicional llamado riesgo de financiamiento. Ambos riesgos hacen referencia a la posibilidad de que no haya ingresos mediante los cuales se puedan cubrir ambos gastos.

Si existen riesgos, entonces el sentido común nos indica que para afrontarlos es

ACTIVOS REQUERIDOS POR LA PLANTA DE QUESO PROVOLONE (\$)

\begin{tabular}{|c|c|c|c|}
\hline Conceptos de activo & Importe total & IVA pagado & Importe fiscal \\
\hline Equipo de proceso & 1015000.00 & 140000.00 & 875000.00 \\
\hline Equipo de transporte & 335240.00 & 46240.00 & 289000.00 \\
\hline Permisos & 13920.00 & 1920.00 & 12000.00 \\
\hline Constitución jurídica & 29580.00 & 4080.00 & 25500.00 \\
\hline Capital neto de trabajo & 451240.00 & 62240.00 & 389000.00 \\
\hline Importe total & 1844980.00 & 254480.00 & 1590500.00 \\
\hline
\end{tabular}


ESTADO DE RESULTADOS PROFORMA CUANDO SÍ HAY CRÉDITO (\$)

\begin{tabular}{|lc|}
\hline Ingresos por ventas & 12329010.00 \\
Costo de lo vendido & 8260584.00 \\
Utilidad bruta & 4068426.00 \\
Gastos de operación & 2569500.00 \\
$\quad$ Gastos erogados en efectivo & 2406000.00 \\
Gastos virtuales & 163500.00 \\
Utilidad de operación & 1498926.00 \\
Gastos financieros & 79525.00 \\
Utilidad antes de impuestos & 1419401.00 \\
Impuesto sobre la renta (30\%) & 425820.30 \\
Participación de los trabajadores en las utilidades (10\%) & 141940.10 \\
Utilidad neta & 851640.60
\end{tabular}

necesario que el negocio genere un rendimiento adicional que compense la toma de dichos riesgos. Y aquí se presenta el siguiente problema: ¿cómo puede generar un mayor rendimiento el mismo proyecto si de entrada los gastos financieros disminuirán las utilidades netas del negocio?

Aquí reside lo interesante del apalancamiento financiero, ya que a simple vista no parece que habrá un mayor rendimiento. Sin embargo, es necesario hacer los cálculos y verificar si en realidad el rendimiento aumenta o disminuye. Así pues, el crédito propiciará que haya algunos cambios en los estados financieros empleados anteriormente.

En efecto, el Estado de Resultados ahora incluye un nuevo rubro denominado "Gastos financieros", así como un nuevo tipo de utilidad denominada "Utilidad antes de impuestos" y podemos notar que tanto el pago de impuestos, el reparto de utilidades a los trabajadores y la utilidad neta han sufrido un deterioro en alguna medida. ¿Es esto positivo? Lo es, desde el punto de vista del dueño del negocio, ya que la inclusión de los gastos financieros permite que el empresario pague menos impuesto sobre la renta, así como un menor reparto de utilidades a los trabajadores. A esto se le conoce como "coraza fiscal" y veremos su efecto sobre el costo del capital a la hora de determinar la tasa de descuento para evaluar el proyecto; tasa que se denomina "Costo ponderado de capital".

Para determinar el nuevo rendimiento, que en este caso, por haber incluido crédito, lo llamaremos "Rendimiento financiero" será necesario determinar un nuevo flujo de efectivo, así como una nueva tasa de descuento que aunque no será necesaria para hacer los cálculos lo será como un punto de referencia para comparar la tasa interna de rendimiento financiera (TIRF), la cual deberá ser mayor que aquella TIRE (TIRF mayor a TIRE) para indicar que el crédito o apalancamiento financiero fue benéfico para el negocio.

Esta nueva tasa de descuento se conoce como "Costo ponderado de capital" y resultó ser de $23.9658 \%$, de acuerdo con el siguiente cuadro: 
DETERMINACIÓN DEL COSTO PONDERADO DE CAPITAL

\begin{tabular}{|c|c|c|c|c|}
\hline Estructura financiera & Importe (\$) & $\begin{array}{l}\text { Costo de } \\
\text { capital }\end{array}$ & $\begin{array}{l}\text { Costo con coraza } \\
\text { fiscal }\end{array}$ & Ponderación \\
\hline Capital social & 1526880.00 & $25.8337 \%$ & $25.8337 \%$ & 394449.60 \\
\hline Crédito refaccionario & 318100.00 & $25.00 \%$ & $15 \%=0.25(0.60)$ & 47715.00 \\
\hline Total & 1844980.00 & & & 442164.60 \\
\hline Costo ponderado de capital & & & $23.9658 \%$ & \\
\hline
\end{tabular}

A continuación se explica la integración de los valores de cada columna.

Luego de identificar la estructura financiera de la primera y segunda columnas, deberán considerarse los costos de cada concepto de la estructura financiera. En el caso del capital social, el costo de capital se refiere al costo de oportunidad que tienen los recursos de los socios, que en este caso es el rendimiento que genera el proyecto cuando no hay crédito, es decir, $25.8337 \%$ anual.

El costo del crédito refaccionario se refiere a la TAE que pagamos por dicho préstamo, dicha tasa es del $25 \%$.

La coraza fiscal se refiere a la deducibilidad fiscal. En el caso del capital social, no existe deducibilidad alguna puesto que el costo de oportunidad, si bien se admite que existe, contablemente no se registra y fiscalmente no es deducible. En cambio, el costo del crédito, esto es, los gastos financieros pagados por el crédito (en este caso $\$ 79$ 525.00) sí son deducibles. A eso se debe que se paguen menos impuestos y un menor reparto de utilidades a los trabajadores.

Si consideramos que los ahorros en el pago de los dos conceptos anteriores (ISR у PTU) son los presentados en el siguiente cuadro (\$31 810.00) y los restamos de la suma de gastos financieros pagados al intermediario financiero (\$79525.00), podríamos decir que la diferencia ( $\$ 47715$ ) representa el pago que efectivamente hicimos al intermediario financiero. Y si dividimos dicho pago entre el importe del crédito (\$47 715.00/\$318 100.00), el resultado será la tasa que efectivamente nos costó el crédito, que para el caso que nos ocupa fue del 15\% anual, y no del $25 \%$. En el cuadro de la ponderación este resultado se obtuvo mediante la siguiente fórmula:

$$
i_{e}=i(1-T)
$$

donde $i_{e}$ es el costo efectivamente pagado por el crédito; $T=(30 \%$ de ISR $+10 \%$ de PTU); $1-T=60 \%$. Luego se tiene: $i_{e}=25 \%$ (1-0.4) e $i_{e}=15 \%$ anual.

\section{DISMINUCIÓN EN EL PAGO DE ISR Y PTU CUANDO HAY CRÉDITO (\$)}

\begin{tabular}{|l|c|c|c|}
\hline Conceptos & No hay crédito & Si hay crédito & Ahorros de ISR Y PTU \\
\hline $\begin{array}{l}\text { Impuesto sobre la renta (30\%) } \\
\begin{array}{l}\text { Participación de los trabajadores } \\
\text { en las utilidades (10\%) }\end{array}\end{array}$ & 607569.90 & 583712.40 & 23857.50 \\
Total & 810093.20 & 194570.80 & 7952.50 \\
\hline
\end{tabular}


ESTADO DE FUENTESY USOS DEL EFECTIVO PROFORMA CUANDO SÍ HAY CRÉDITO (\$)

\begin{tabular}{|c|c|c|}
\hline \multicolumn{3}{|l|}{ Fuentes de efectivo } \\
\hline Capital social & 1526880.00 & \\
\hline Crédito refaccionario & 318100.00 & \\
\hline Utilidades netas & & 851640.60 \\
\hline Gastos virtuales & & 163500.00 \\
\hline IVA acreditable & & 254480.00 \\
\hline Valor de rescate & & 1004250.00 \\
\hline Total de fuentes de efectivo & 1844980.00 & 2273870.60 \\
\hline \multicolumn{3}{|l|}{ Usos del efectivo } \\
\hline Activos fijos & 1164000.00 & \\
\hline Activos diferidos & 37500.00 & \\
\hline Capital neto de trabajo & 389000.00 & \\
\hline IVA pagado & 254480.00 & \\
\hline Pago del crédito refaccionario & & 318100.00 \\
\hline Total de usos de efectivo & 1844980.00 & 318100.00 \\
\hline Flujo neto de efectivo & 0.00 & 1955770.60 \\
\hline
\end{tabular}

La ponderación de la última columna se refiere al producto de los importes de cada elemento de la estructura financiera por el costo con coraza fiscal.

Finalmente, el costo ponderado de capital es el cociente de la suma de la última columna (\$706 080.96) y el importe total empleado en la adquisición de los activos ( $\$ 1$ 844 980.00), este cociente fue de $23.9658 \%$. Dicha cifra es menor que el costo de oportunidad que tiene Gianluca cuando invierte sus propios recursos, lo cual nos hace suponer que la nueva rentabilidad (cuando hay crédito) deberá ser mayor que el costo de oportunidad. ¿Tiene sentido? La pregunta es relevante, especialmente porque ya vimos que la utilidad neta cuando hay crédito es menor que la que se tiene cuando no hay crédito, y observando el Estado de Fuentes y Usos del Efectivo notamos que también el flujo neto de efectivo es menor que cuando no hay crédito.
Contestaremos esta pregunta posteriormente, por ahora continuemos determinando el nuevo flujo neto de efectivo, para lo cual se requiere nuevamente la elaboración de otro Estado de Fuentes y Usos del Efectivo. Este estado financiero, como en el caso del Estado de Resultados, también incluirá nuevos rubros. Por el lado de las fuentes de efectivo ahora hay un nuevo rubro denominado "Crédito refaccionario" y por el lado de los usos hay otro nuevo rubro denominado "Pago del crédito refaccionario".

Finalmente, con el nuevo flujo de efectivo calcularemos la TIRF para compararla con la TIRE a fin de ver cuál de las dos es mayor y saber, de esta manera, si el apalancamiento financiero incrementa o disminuye el rendimiento del capital social.

Como puede apreciarse en el siguiente cuadro, el rendimiento financiero fue del $28.09 \%$ anual y es mayor que el rendimiento sin crédito, esto es, mayor que $25.8337 \%$ anual. 
CÁLCULO DE LA TASA INTERNA DE RENDIMIENTO FINANCIERA

\begin{tabular}{|l|c|c|r|r|c|}
\hline Años & Fne & FA al 28\% & Fne actualizado & FA al 29\% & Fne \\
\hline 0 & -1526880.00 & 1.0000 & -1526880.00 & 1.0000 & -1526880.00 \\
1 & 1955770.60 & 0.7813 & 1527945.80 & 0.7752 & 1516101.20 \\
Valor actual neto & & & 1065.80 & & -10778.80 \\
\hline
\end{tabular}

La TIRF se calculó mediante la fórmula:

$$
\text { TIRF }=28 \%+\frac{(29-28)(1065.80)}{(1065.80+10778.80)}
$$$$
\text { TIRF }=28 \%+0.09 \%
$$

De esta manera, podemos concluir que el apalancamiento financiero de la fábrica de queso provolone es benéfico, ya que genera un rendimiento adicional de 2.2563 puntos porcentuales, lo cual equivale a decir que hubo un incremento en la rentabilidad del $8.73 \%$, siendo este aumento el premio que el proyecto paga por asumir el riesgo financiero. Es decir, si el apalancamiento financiero representa un riesgo financiero, adicional al riesgo operativo, es obligado que también genere un premio por asumir dicho riesgo.

Los resultados presentados hasta aquí llevan a preguntarnos: ¿y qué pasará si se incrementa el porcentaje de deuda o crédito que participa en la estructura financiera, digamos si en lugar de $20 \%$ el porcentaje sube a $40 \%, 60 \%$ o $80 \%$ ? ¿Se mantiene el premio por el riesgo financiero? ¿Aumenta o disminuye?

Calculando los nuevos estados financieros, los flujos de efectivo y la tasa interna de rendimiento financiero, los resultados se presentan en el cuadro de abajo.

\section{CONCLUSIONES}

De los resultados anteriores puede concluirse que cuando el costo de capital del financiamiento es menor que el rendimiento económico del proyecto se puede esperar que el rendimiento financiero del mismo sea mayor al rendimiento económico, y seguirá una tendencia alcista en función directa del grado en el que se emplee dicho apalancamiento. Es decir, que siempre esperaremos que a mayor porcentaje de palanca financiera mayor será el rendimiento financiero. Entendiéndose también que a mayor apalancamiento mayor riesgo financiero, pero también un mayor premio por asumir dicho riesgo.

Se omitieron los cálculos referidos a los demás porcentajes de palanca financiera ( $40 \%, 60 \%$ y $80 \%)$ en aras de compactar este documento y no alargarlo innecesariamente. Pero los lectores podrían verificar los resultados haciendo el ejercicio de calcularlos por sí mismos, esto hará, sin duda, que este material resulte varias veces más provechoso.

EFECTO DEL APALANCAMIENTO FINANCIERO SOBRE EL RENDIMIENTO DEL CAPITAL SOCIAL

\begin{tabular}{|c|l|l|l|r|}
\hline Palanca financiera (\%) & TIRE (\%) & TIRF (\%) & \multicolumn{2}{|c|}{ Incremento absoluto (\%) } \\
\hline 0 & 25.8337 & & & \\
20 & 25.8337 & 28.09 & 2.2563 puntos & 8.73 \\
40 & 25.8337 & 31.5358 & 5.7021 puntos & 22.07 \\
60 & 25.8337 & 37.4407 & 11.6070 puntos & 44.93 \\
80 & 25.8337 & 49.9055 & 24.0718 puntos & 93.17 \\
\hline
\end{tabular}

